TITLE:

\title{
Differential effects of fingolimod on B-cell populations in multiple sclerosis(Dissertation_全文)
}

$\operatorname{AUTHOR}(S)$ :

Nakamura, Masakazu

\section{CITATION:}

Nakamura, Masakazu. Differential effects of fingolimod on B-cell populations in multiple sclerosis. 京都大学, 2014, 博士(医学)

ISSUE DATE:

2014-11-25

URL:

https://doi.org/10.14989/doctor.r12871

RIGHT: 


\section{Multiple Sclerosis Journal}

http://msj.sagepub.com/

Differential effects of fingolimod on B-cell populations in multiple sclerosis

Masakazu Nakamura, Takako Matsuoka, Norio Chihara, Sachiko Miyake, Wakiro Sato, Manabu Araki, Tomoko Okamoto,

Youwei Lin, Masafumi Ogawa, Miho Murata, Toshimasa Aranami and Takashi Yamamura

Mult Scler published online 13 February 2014

DOI: $10.1177 / 1352458514523496$

The online version of this article can be found at:

http://msj.sagepub.com/content/early/2014/02/18/1352458514523496.citation

\section{Published by: \\ (\$) SAGE}

http://www.sagepublications.com

Additional services and information for Multiple Sclerosis Journal can be found at:

Email Alerts: http://msj.sagepub.com/cgi/alerts

Subscriptions: http://msj.sagepub.com/subscriptions

Reprints: http://www.sagepub.com/journalsReprints.nav

Permissions: http://www.sagepub.com/journalsPermissions.nav

>> OnlineFirst Version of Record - Feb 18, 2014

OnlineFirst Version of Record - Feb 13, 2014

What is This? 


\section{Differential effects of fingolimod on B-cell populations in multiple sclerosis}

\author{
Masakazu Nakamura',2, Takako Matsuoka', Norio Chihara', \\ Sachiko Miyake ${ }^{1,3}$, Wakiro Sato ${ }^{3,4}$, Manabu Araki ${ }^{3}$, \\ Tomoko Okamoto ${ }^{3,4}$, Youwei Lin 1,3,4, Masafumi Ogawa ${ }^{3,4}$, \\ Miho Murata ${ }^{4}$, Toshimasa Aranami ${ }^{1,3}$ and Takashi Yamamura ${ }^{1,3}$
}

Multiple Sclerosis Journal

I-10

(C) The Author(s) 2014

Reprints and permissions:

sagepub.co.uk/journalsPermissions.nav DOI: 10.1 I 177//3524585/4523496 msj.sagepub.com

(S)AGE

\begin{abstract}
Background: Fingolimod is an oral drug approved for multiple sclerosis (MS) with an ability to trap central memory $\mathrm{T}$ cells in secondary lymphoid tissues; however, its variable effectiveness in individual patients indicates the need to evaluate its effects on other lymphoid cells.

Objective: To clarify the effects of fingolimod on B-cell populations in patients with MS.

Methods: We analysed blood samples from 9 fingolimod-treated and 19 control patients with MS by flow cytometry, to determine the frequencies and activation states of naive B cells, memory B cells, and plasmablasts.

Results: The frequencies of each B-cell population in peripheral blood mononuclear cells (PBMC) were greatly reduced 2 weeks after starting fingolimod treatment. Detailed analysis revealed a significant reduction in activated memory $B$ cells (CD38 int-high), particularly those expressing Ki-67, a marker of cell proliferation. Also, we noted an increased proportion of activated plasmablasts $\left(\mathrm{CDI} 38^{+}\right)$among whole plasmablasts, in the patients treated with fingolimod.

Conclusions: The marked reduction of $\mathrm{Ki}-67^{+}$memory $\mathrm{B}$ cells may be directly linked with the effectiveness of fingolimod in treating MS. In contrast, the relative resistance of $\mathrm{CDI} 38^{+}$plasmablasts to fingolimod may be of relevance for understanding the differential effectiveness of fingolimod in individual patients.
\end{abstract}

\title{
Keywords
}

B cells, CD38, CDI38, fingolimod, memory B cell, multiple sclerosis, plasmablast, proliferation, resistance, sphingosine I-phosphate receptor I

Date received: 5 September 20I3; accepted: 16 January 2014

\section{Introduction}

It is currently assumed that a large proportion of autoreactive $\mathrm{T}$ cells in multiple sclerosis (MS) is derived from a pool of $\mathrm{CCR} 7^{+}$central memory $\mathrm{T}$ cells that are passing through the secondary lymphoid tissues (SLT). ${ }^{1}$ Accordingly, egress of the T cells from the SLT represents a key process in MS pathogenesis. This process follows a rule of chemotaxis, in which the sphingosine 1-phosphate (S1P) receptor 1 (S1P1) expressed by lymphocytes is critically involved. ${ }^{2}$ Fingolimod, an oral drug for treating relapsing-remitting MS (RRMS), serves as a functional antagonist for S1P1: Fingolimod induces internalisation and degradation of S1P1 in lymphocytes, causing the lymphocytes to lose the ability to respond to S1P and consequently, to become trapped in the SLT. ${ }^{3}$ Analysis of large cohorts of patients with RRMS demonstrate the overall effectiveness of fingolimod in reducing the annualised relapse rate (ARR), as well as the appearance of new brain lesions in the patients' magnetic resonance imaging (MRI) scans. ${ }^{4,5}$
The number of central memory interleukin 17-producing $\mathrm{CD}^{+} \mathrm{T}$ cells (Th17 cells) is reduced in the peripheral blood of fingolimod-treated patients. This is now being interpreted as a major mechanism of drug action; ${ }^{6}$ however, fingolimod is not able to prevent relapses nor exhibit

\footnotetext{
'Department of Immunology, National Institute of Neuroscience, National Centre of Neurology and Psychiatry (NCNP), Tokyo, Japan. ${ }^{2}$ Department of Neurology, Graduate School of Medicine, Kyoto University, Kyoto, Japan.

3Multiple Sclerosis Centre, National Centre Hospital, NCNP, Tokyo, Japan. ${ }^{4}$ Department of Neurology, National Centre Hospital, NCNP, Tokyo, Japan.

Corresponding author:

Takashi Yamamura, Department of Immunology, National Institute of Neuroscience, National Centre of Neurology and Psychiatry, 4-I-I Ogawahigashi, Kodaira, Tokyo 187-8502, Japan.

Email: yamamura@ncnp.go.jp
} 
Table I. Clinical data of the patients in this study.

\begin{tabular}{|c|c|c|c|c|c|c|c|}
\hline Patient & Gender & Age (years) & Duration (years) & $\begin{array}{l}\text { Relapse frequency } \\
\text { (last } 2 \text { yrs) }\end{array}$ & EDSS & DMT before initiation of fingolimod & Complications \\
\hline I & M & 34 & 7 & 5 & 1.5 & IFN $\beta$ Ia + PSL & Asthma \\
\hline 2 & M & 43 & 6 & 2 & 2.5 & PSL & Graves' disease \\
\hline 3 & M & 39 & 5 & I & 3.5 & None & Depression \\
\hline 4 & M & 41 & 13 & I & 3.5 & IFN $\beta$ Ib & None \\
\hline 5 & M & 29 & 2 & 3 & 2.0 & IFN $\beta$ Ib & Pectus excavatum \\
\hline 6 & $\mathrm{~F}$ & 41 & 24 & 6 & 3.5 & IFN $\beta \mathrm{Ib} \rightarrow \mathrm{GA} \rightarrow$ Dex & Depression \\
\hline 7 & M & 56 & 16 & 2 & 5.5 & $\begin{array}{l}\mathrm{IFN} \beta \mathrm{I} \mathrm{b} \rightarrow \mathrm{IFN} \beta \mathrm{I} b+\mathrm{PSL} \rightarrow \\
\mathrm{IFN} \beta \mathrm{I} \mathrm{a}+\mathrm{AZP}\end{array}$ & Osteoporosis \\
\hline 8 & M & 41 & 9 & 2 & 4.0 & IFN $\beta$ Ib $\rightarrow$ IFN $\beta$ Ia & Depression \\
\hline $\begin{array}{l}9 \\
\text { mean } \pm \\
S D\end{array}$ & M & $\begin{array}{l}60 \\
42.7 \pm 9.8\end{array}$ & $\begin{array}{l}20 \\
11.3 \pm 7.4\end{array}$ & $2.5 \pm 1.8$ & $\begin{array}{l}3.5 \\
3.3 \pm 1.2\end{array}$ & $\mathrm{AZP} \rightarrow \mathrm{MZR} \rightarrow \mathrm{IFN} \beta \mathrm{Ib}$ & None \\
\hline
\end{tabular}

AZP: Azathioprine; Dex: dexamethasone; DMT: disease-modifying treatment; EDSS: Expanded Disability Status Scale; F: female; GA: glatiramer acetate; IFN: interferon; M: male; MZR: mizoribine; PSL: prednisolone.

appreciable effectiveness in all patients. In fact, recent case reports document the presence of fingolimod-treated MS patients who have developed tumefactive brain lesions, after receiving fingolimod. ${ }^{7-10}$ Moreover, clinical worsening accompanied by large brain lesions is described in patients with neuromyelitis optica (NMO), within months of starting fingolimod. ${ }^{11,12}$ Our current understanding of fingolimod-related biology therefore remains incomplete, particularly regarding differential effectiveness in individual patients.

Not only the presence of clonally-expanded B cells in the central nervous system (CNS), ${ }^{13,14}$ but the efficacy of the anti-CD20 monoclonal antibody $(\mathrm{mAb})$ rituximab $^{15}$ rationally indicates the involvement of $\mathrm{B}$ cells in the pathogenesis of MS. Therefore, B-cell migration can serve as a therapeutic target in MS, so we were prompted to investigate whether inhibition of B-cell migration may explain the differential effectiveness of fingolimod. Because the effects of fingolimod on B cells in MS have not been fully characterised, ${ }^{16}$ we analysed the alterations of B-cell populations in fingolimod-treated RRMS patients by flow cytometry, measuring the frequencies and activation states of their peripheral blood B-cell populations.

\section{Materials and methods}

\section{Patients and sample collection}

The following subjects were enrolled in the Multiple Sclerosis Clinic of the National Centre of Neurology and Psychiatry (NCNP) in Japan:

(a) Fingolimod-naïve patients with RRMS ( $n=9)$;

(b) RRMS patients who were treated with other diseasemodifying treatments (DMTs) or corticosteroids $(n=$ 19); and

(c) Healthy donors $(n=3)$.
All MS patients fulfilled the revised McDonald criteria. ${ }^{17}$ Fingolimod ( $0.5 \mathrm{mg}$ once/day) was administered to nine fingolimod-naïve patients. These patient's blood samples were collected before and 2 weeks after initiating fingolimod therapy. Most of these patients discontinued other DMTs at least 2 weeks before entry into the study, due to non-responsiveness to their DMT treatment or due to adverse events. The absence of serum anti-aquaporin 4 (AQP4)-Ab was confirmed by cell-based assays. ${ }^{18,19}$ Upon MRI, no patient showed longitudinally-extensive spinal cord lesions extending over three or more vertebrae. The clinical data of these nine patients are summarised in Table 1.

Control blood samples were collected from 19 patients with RRMS (mean age \pm SD: $41.8 \pm 13.8$ years; female:male ratio: 15:4) who had not been exposed to fingolimod before nor during the study. The three healthy donors were males (mean age \pm SD: $40.0 \pm 3.6$ years). This study was approved by the Ethics Committee of the NCNP. We obtained written informed consent from all subjects.

\section{Reagents}

The following fluorescence- or biotin-labelled mAbs were used: anti-CD19-allophycocyanin (APC)-cyanine 7 (Cy7), anti-CD27-V500 and anti-CD27-phycoerythrin (PE)-Cy7 (BD Biosciences, San Jose, CA, USA); anti-CD180-PE and anti-CCR7-fluorescein isothiocyanate (FITC) (BD Pharmingen, San Jose, CA, USA); anti-CD38-FITC, antiCD3-FITC and mouse IgG1-FITC (Beckman Coulter,

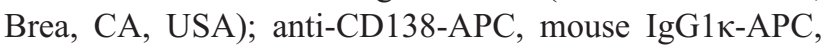
anti-HLA-DR-Pacific Blue, mouse IgG2Aк-Pacific Blue, anti-CD183 (CXCR3)-peridinin-chlorophyll-protein (PerCp)-cyanine 5.5 (Cy5.5), mouse IgG1к-PerCp-Cy5.5, anti-CD38-APC, anti-CD38-PerCp-Cy5.5, anti-CD14Pacific Blue, anti-Ki-67-Brilliant Violet, mouse IgG1кBrilliant Violet and streptavidin-PE-Cy7 (BioLegend, San 
Diego, CA, USA); and anti-CXCR4-biotin and mouse IgG2A-biotin (R\&D Systems, Minneapolis, MN, USA).

\section{Cell preparation and flow cytometry}

Peripheral blood mononuclear cells (PBMC) were isolated by density gradient centrifugation, using Ficoll-Paque Plus (GE Healthcare Bioscience, Oakville, ON, Canada). B-cell populations were defined in reference to our previous paper, ${ }^{19}$ as follows: total B cells, CD19 ${ }^{+}$; naïve B cells (nBs), $\mathrm{CD} 19^{+} \mathrm{CD} 27^{-}$; memory B cells (mBs), $\mathrm{CD} 19^{+} \mathrm{CD} 27^{+} \mathrm{CD} 180^{+}$; and plasmablasts (PBs), $\mathrm{CD} 19^{+} \mathrm{CD} 27^{+} \mathrm{CD} 180^{-} \mathrm{CD} 38^{\text {high }}$.

To evaluate the frequency and activation state of each B-cell population, $\mathrm{PBMC}$ were stained with anti-CD19-APCCy7, anti-CD27-V500, anti-CD38-FITC, anti-CD180-PE, anti-CD138-APC, anti-CXCR3-PerCp-Cy5.5, anti-CXCR4biotin, streptavidin-PE-Cy7 and anti-HLA-DR-Pacific Blue. To assess the expression of CCR7 in each $B$ cell population, PBMC were stained with anti-CD19-APC-Cy7, anti-CD27PE-Cy7, anti-CD38-APC, anti-CD180-PE and anti-CCR7FITC.

For examining Ki-67 expression in each B-cell population, PBMC were stained with anti-CD19-APC-Cy7, anti-CD27-PE-Cy7, anti-CD38-PerCp-Cy5.5, anti-CD180$\mathrm{PE}$ and anti-CD138-APC, then fixed in phosphate-buffered saline (PBS) containing 2\% paraformaldehyde and permeabilised with $0.1 \%$ saponin. Subsequently, these cells were stained with anti-Ki-67-Brilliant Violet. We used the appropriate isotype control antibodies as negative controls for each staining. At the end of the incubation, the cells were washed and resuspended in PBS supplemented with $0.5 \%$ bovine serum albumin (BSA) and analysed by FACS Canto II (BD Biosciences), according to the manufacturer's instructions.

\section{Cell sorting}

PBMC were labelled with CD3 and CD14 microbeads (Miltenyi Biotec, Bergisch Gladbach, Germany) and then separated into positive and negative fractions by AutoMACS (Miltenyi Biotec). The positive fraction was stained with anti-CD3-FITC and anti-CD14-Pacific Blue, whereas the negative fraction was stained with anti-CD19-APCCy7, anti-CD27-PE-Cy7, anti-CD38-APC and anti-CD180$\mathrm{PE}$. Each positive and negative fraction was sorted into $\mathrm{CD}^{+} \mathrm{T}$ cells and $\mathrm{CD} 14^{+}$monocytes, or into $\mathrm{nBs}, \mathrm{mBs}$ and PBs by a FACS Aria II cell sorter (BD Biosciences). The purity of the sorted cells was $>95 \%$.

\section{Quantitative real-time PCR}

Messenger ribonucleic acid (mRNA) was prepared from the sorted cells using the RNeasy Kit (Qiagen, Tokyo, Japan), further treated with DNase using the RNase-Free DNase Set (Qiagen), and reverse-transcribed to complementary DNA (cDNA) using the cDNA Synthesis Kit (Takara Bio, Shiga, Japan). We performed polymerase chain reaction (PCR) using iQ SYBR Green Supermix (Takara Bio) on a LightCycler (Roche Diagnostics, Indianapolis, IN, USA). RNA levels were normalised to endogenous $\beta$-actin (ACTB) for each sample. The following primers were used: S1P1 forward, CGAGAGCACTACGCAGTCAG; and S1P1 reverse, AGAGCCTTCACTGGCTTCAG.

\section{Data analysis and statistics}

We used Diva software (BD Biosciences) to analyse our flow cytometry data. We performed the statistical analysis with Prism software (GraphPad Software, San Diego, CA, USA). Paired or unpaired $t$-tests were used once the normality of the data was confirmed by the KolmogorovSmirnov test. Otherwise, the Wilcoxon signed-rank test or the Mann-Whitney $U$-test was used, as appropriate. Oneway analysis of variance (ANOVA) was used to compare data from more than two groups. If the one-way ANOVA was significant, we performed post hoc pairwise comparisons using Tukey's test. A $p$ value $<0.05$ was considered statistically significant.

\section{Results}

\section{B-cell populations express SIPI mRNA}

First, we used flow cytometry to examine S1P1 expression on the surfaces of the B-cell populations; however, surface S1P1 was hardly detected (data not shown). This is probably because of its internalisation following S1P binding. In support of this, it is known that S1P is abundantly present in peripheral blood. ${ }^{2}$ Thus, we measured S1P1 mRNA in purified lymphocyte populations from the PBMCs of three healthy donors. Each B-cell population was identified by flow cytometry, as shown in Figure 1(a). We found that comparable levels of S1P1 mRNA were expressed in $\mathrm{T}$ cells, $\mathrm{nBs}$ and $\mathrm{mBs}$. In comparison, $\mathrm{PBs}$ expressed a significantly lower level of S1P1, and S1P1 expression in monocytes was virtually absent (Figure 1(b)). Of note, a lower S1P1 expression by PBs, as compared with other B cell populations, is also described in mice. ${ }^{20,21}$ These S1P1 mRNA expression profiles suggested that not only $\mathrm{T}$ cells, but B-cell migration, could also be influenced by fingolimod.

Next, we measured the frequencies of the B-cell populations in the PBMCs from nine patients with RRMS, before and 2 weeks after starting fingolimod. Results of flow cytometry showed that the frequencies of $\mathrm{nBs}, \mathrm{mBs}$ and PBs among PBMCs were significantly decreased after initiating fingolimod treatment (Figure 1(c)). We confirmed that the absolute numbers of each population in the peripheral blood were also significantly decreased after starting fingolimod (Figure 1(d)). The mean decrease rate \pm SD of each cell population was calculated based on the absolute cell number, giving the following results: total B cells, 87.6 $\pm 5.8 \%$; $\mathrm{nBs}, 88.1 \pm 6.0 \%$; $\mathrm{mBs}, 85.4 \pm 9.1 \%$ and $\mathrm{PBs}, 89.8$ 
(a)

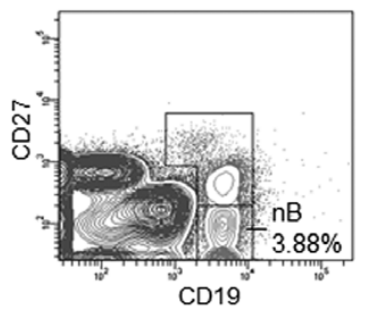

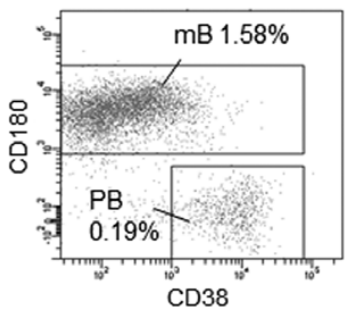

(b)

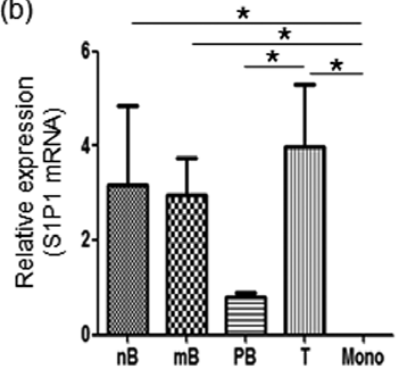

(c)

$\mathrm{CD}^{2} 9^{+} \mathrm{B}$

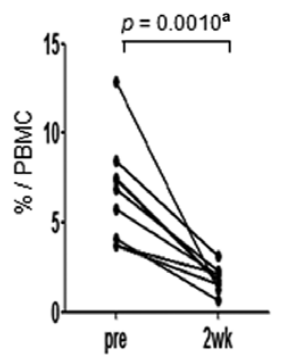

(d)

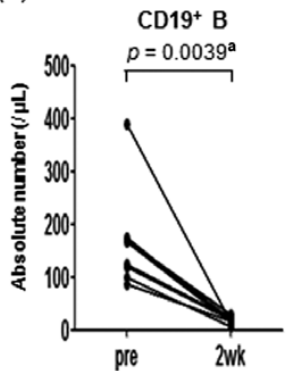

(e)

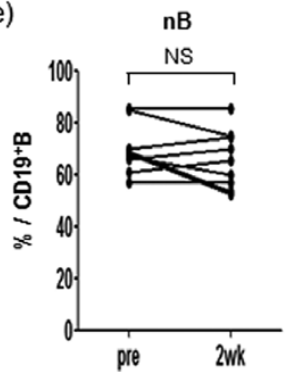

nB

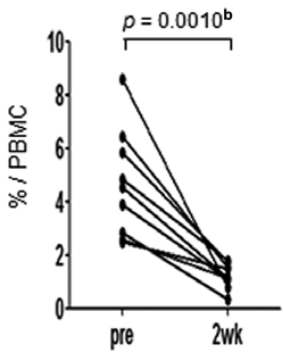

$\mathrm{nB}$
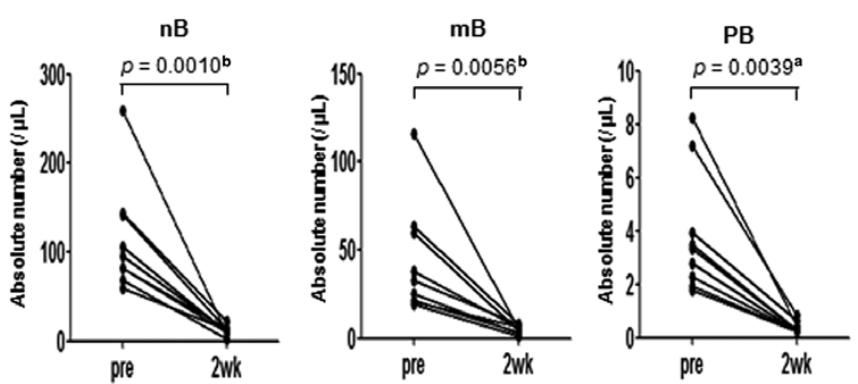

PB
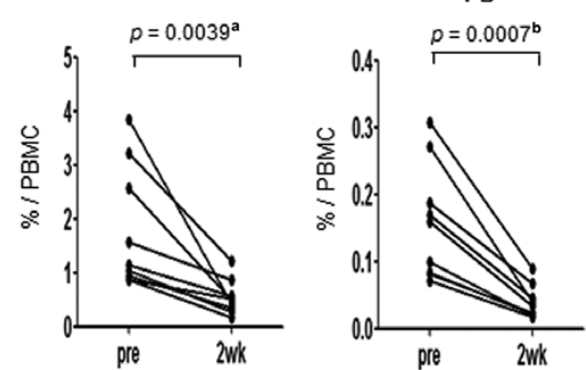
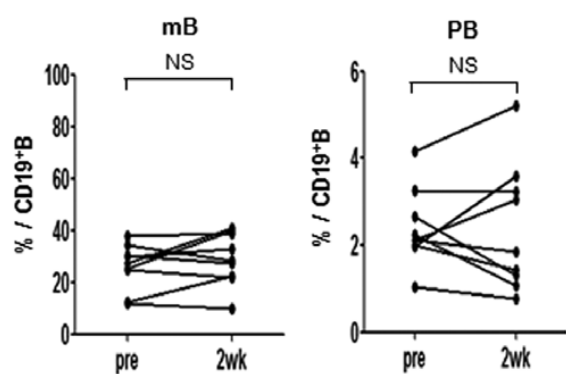

Figure I. Frequency and absolute number of each B-cell population found in peripheral blood from MS patients.

(a)Representative flow cytometry scheme to analyse B-cell populations in PBMC. The PBMC were simultaneously stained with fluorescenceconjugated anti-CD 19, -CD27, -CD38 and - CD $180 \mathrm{mAbs}$. The gate for CD $19^{+} \mathrm{CD} 27^{-} \mathrm{nBs}$ is shown in the left panel. The CDI9+CD27 fraction partitioned in the left panel was analysed for CDI80 and CD38 expression to specify CDI80+ cells (mBs), and for CDI80-CD38high cells (PBs) in the right panel. Values represent frequencies of B-cell populations in PBMC. Total CDI9+ B cell counts were calculated by summing the frequencies of the partitioned populations in the left panel. (b) Each B-cell population, CD3 ${ }^{+} \mathrm{T}$ cells and $\mathrm{CDI} 4^{+}$monocytes in PBMCs from three healthy donors were sorted by FACS, and SIPI mRNA expression levels were determined by quantitative RT-PCR. Data were normalised to the amount of ACTB for each sample. Data are represented as mean relative expression \pm SD. *p $<0.05$ by one-way ANOVA and post hoc Tukey's test. (c), (d), and (e) Data shown are the frequencies of B-cell populations in PBMC (c), the absolute numbers of B cell populations in peripheral blood (d) and the frequencies of B-cell populations in CDI9+ B cells (e) from nine patients with MS before (pre) and 2 weeks after ( 2 wk) initiating fingolimod. Data from the same patients are connected with lines.

$p^{a}<0.05$ by Wilcoxon signed-rank test.

$p^{b}<0.05$ by paired $t$-test.

ACTB: endogenous beta actin; ANOVA: analysis of variance; FACS: Fluorescence-activated cell sorting; mAbs: monoclonal antibodies; mBs: memory B cells; mono: monocytes; mRNA: messenger ribonucleic acid; MS: multiple sclerosis; $n B s:$ naïve B cells; NS: not statistically significant; PBMC: peripheral blood mononuclear cells; PBs: plasmablasts; pre: before treatment; RT-PCR: reverse transcriptase - polymer chain reaction; SIPI: sphingosine I phosphate receptor I; T: T cells; 2 wk: 2 weeks after treatment initiation 
$\pm 3.3 \%$. Thus, all B-cell populations decreased at similar rates, regardless of their S1P1 expression levels. We also noticed that reduction of the B-cell populations did not correlate with CCR7 expression (a large proportion of $\mathrm{nBs}$ and $\mathrm{mBs}$ expresses CCR7, whereas only a small percentage of PBs expresses CCR7 (Supplementary Figure 1)). Consistently, the frequency of each B-cell population within $\mathrm{CD} 19^{+} \mathrm{B}$ cells was not significantly altered in the fingolimod-treated patients (Figure 1(e)).

\section{CD38 int_ and CD38 $8^{\text {high-activated memory } B}$ cells are preferentially decreased in fingolimod- treated patients}

We next assessed $\mathrm{mBs}$, which are assumed to play an important role in MS. ${ }^{22,23}$ To evaluate the effects of fingolimod on the activation state of $\mathrm{mBs}$, we first analysed CD38 expression of $\mathrm{mBs}$ in the nine patients, before and after initiating fingolimod. CD38 is a marker that is upregulated upon B-cell activation. ${ }^{24} \mathrm{We}$ found that $\mathrm{mBs}$ could be classified into three subpopulations according to CD38 expression levels (CD38 low, $\mathrm{CD} 38^{\text {int }}$ and $\left.\mathrm{CD} 38^{\text {high }}\right)$. Notably, frequencies of $\mathrm{CD} 38^{\text {int }}$ and $\mathrm{CD} 38^{\text {high }} \mathrm{mBs}$ were significantly decreased 2 weeks after initiating fingolimod, whereas the frequency of the $\mathrm{CD} 38^{\text {low }}$ subpopulation became significantly increased (Figure 2(a) and (b)).

We further examined the expression of another activation marker, HLA-DR, within the CD38 $8^{\text {low }}, \mathrm{CD} 38^{\text {int }}$ and $\mathrm{CD} 38^{\text {high }} \mathrm{mB}$ subpopulations. We found that the $\mathrm{CD} 38^{\text {high }}$ subpopulation expressed a significantly higher level of HLA-DR, compared with the CD38 low $\mathrm{mB}$ population, as assessed by mean fluorescence intensities (MFIs) (Figure 2(c) and (d)). Although not statistically significant, HLA-DR expression in the CD38 $8^{\text {int }}$ subpopulation was intermediate, compared with that in the $\mathrm{CD} 38^{\text {low }} \mathrm{mB}$ subpopulation. We also found that the MFIs of forward scatter (FSC), which reflects cell size, were significantly higher in the $\mathrm{CD} 38^{\text {high }}$ subpopulation, compared with the CD38 low and CD38 int subpopulations (Figure 2(c) and (d)). These findings suggest that $\mathrm{CD} 38^{\text {high }} \mathrm{mBs}$ may contain a larger number of recently-activated blastic cells.

\section{Fingolimod reduced $\mathrm{Ki}-67^{+}$recently-activated memory $B$ cells in peripheral blood}

The nuclear antigen Ki-67 is exclusively expressed in the active stages of the cell cycle (G1, S, G2 and M phases), ${ }^{25}$ and $\mathrm{Ki}-67^{+}$circulating immune cells are considered to be recently activated cells that have just egressed from the SLT. To clarify whether CD $38^{\text {high }}$ and CD $38^{\text {int }} \mathrm{mB}$ subpopulations are enriched for recently-activated cells, we examined the frequency of $\mathrm{Ki}-67^{+}$cells in each $\mathrm{mB}$ subpopulation, in the six MS patients who were not treated with fingolimod. This analysis revealed that $\mathrm{CD} 38^{\text {high }} \mathrm{mBs}$ contained a significantly higher frequency of $\mathrm{Ki}-67^{+}$cells than did $\mathrm{CD} 38^{\text {low }}$ and $\mathrm{CD} 38^{\text {int }} \mathrm{mBs}$, and that $\mathrm{CD} 38^{\text {int }} \mathrm{mBs}$ were likely to contain a higher frequency of $\mathrm{Ki}-67^{+}$cells than the CD38 $8^{\text {low }} \mathrm{mBs}$ (Figure 3(a) and (b)). In addition, we compared the frequency of $\mathrm{Ki}-67^{+}$cells in each $\mathrm{mB}$ subpopulation, between fingolimod-treated $(n=5)$ and -untreated control patients $(n=6)$, and found that $\mathrm{CD} 38^{\text {int }}$ and $\mathrm{CD} 38^{\text {high }}$ $\mathrm{mBs}$ of the fingolimod-treated patients contained a significantly lower percentage of $\mathrm{Ki}-67^{+}$cells compared with those of the untreated patients (Figure 3(c)). These findings suggest that recently activated $\mathrm{mBs}$ are enriched in CD38 int and $\mathrm{CD} 38^{\text {high }}$ subpopulations and that fingolimod efficiently blocks the egress of these cells from the SLT into the peripheral circulation.

\section{The $\mathrm{CD} / 38^{+}$subpopulation in plasmablasts is relatively resistant to fingolimod}

Finally, we analysed alterations of PBs by fingolimod in more detail. As PBs serve as migratory B cells that produce pathogenic autoantibody directed against AQP4, ${ }^{19}$ their role in the antibody-mediated pathology is being considered also in the pathogenesis of MS. Notably, CD138 expression appears to separate PB subpopulations that could become differentially altered during the inflammatory process. In fact, $\mathrm{CD} 138^{+} \mathrm{PBs}$ have a higher potential to migrate to inflamed tissues than CD138- $\mathrm{PBs} .{ }^{26}$ Moreover, as has recently been reported by us, CD138 ${ }^{+} \mathrm{HLA}^{-\mathrm{DR}^{+} \mathrm{PBs}}$ are selectively enriched in the cerebrospinal fluid (CSF) during relapse of $\mathrm{NMO}$, and the $\mathrm{CD} 138^{+} \mathrm{HLA}-\mathrm{DR}^{+} \mathrm{PBs}$ migrating to the CSF express CXCR3. ${ }^{27}$ Therefore, we compared the frequencies of $\mathrm{CD} 138^{+}$cells in PBs, as well as their expression of HLA-DR and CXCR3, before and after fingolimod treatment.

We found that the frequencies of $\mathrm{CD} 138^{+} \mathrm{PBs}$ among total PBs were significantly increased after fingolimod initiation (Figure 4(a) and (b)); however, the absolute numbers of both subpopulations decreased, implying that $\mathrm{CD} 138^{+} \mathrm{PBs}$ are relatively resistant to fingolimod, compared with CD138- PBs (Supplementary Figure 2(a) and (b)). After initiating fingolimod, CD138- PBs showed lower expression of HLA-DR, whereas the percentages of $\mathrm{CXCR}^{+}$cells remained unchanged (Figure 4(c) - (e)). In contrast, fingolimod treatment did not significantly reduce the expression level of HLA-DR among CD138 $8^{+}$PBs. More interestingly, $\mathrm{CD} 138^{+} \mathrm{PBs}$ became more enriched with $\mathrm{CXCR}^{+}$cells after initiating fingolimod (Figure 4(c) (e)). The definition of $\mathrm{PBs}$ as $\mathrm{CD} 19^{+} \mathrm{CD} 27^{+} \mathrm{CD} 180^{-} \mathrm{CD} 38^{\text {high }}$ cells in this study was modified to efficiently specify autoantibody-producing cells, ${ }^{19}$ however, adopting a more commonly used definition of $\mathrm{PBs}$ as $\mathrm{CD} 19^{+} \mathrm{CD} 27^{+} \mathrm{CD} 38^{\text {high }}$ cells did not alter the results (Supplementary Figure 3(a) $-(\mathrm{e}))$.

\section{Discussion}

Previous studies show that fingolimod markedly decreases the number of $\mathrm{T}$ and $\mathrm{B}$ cells in the peripheral blood, without 


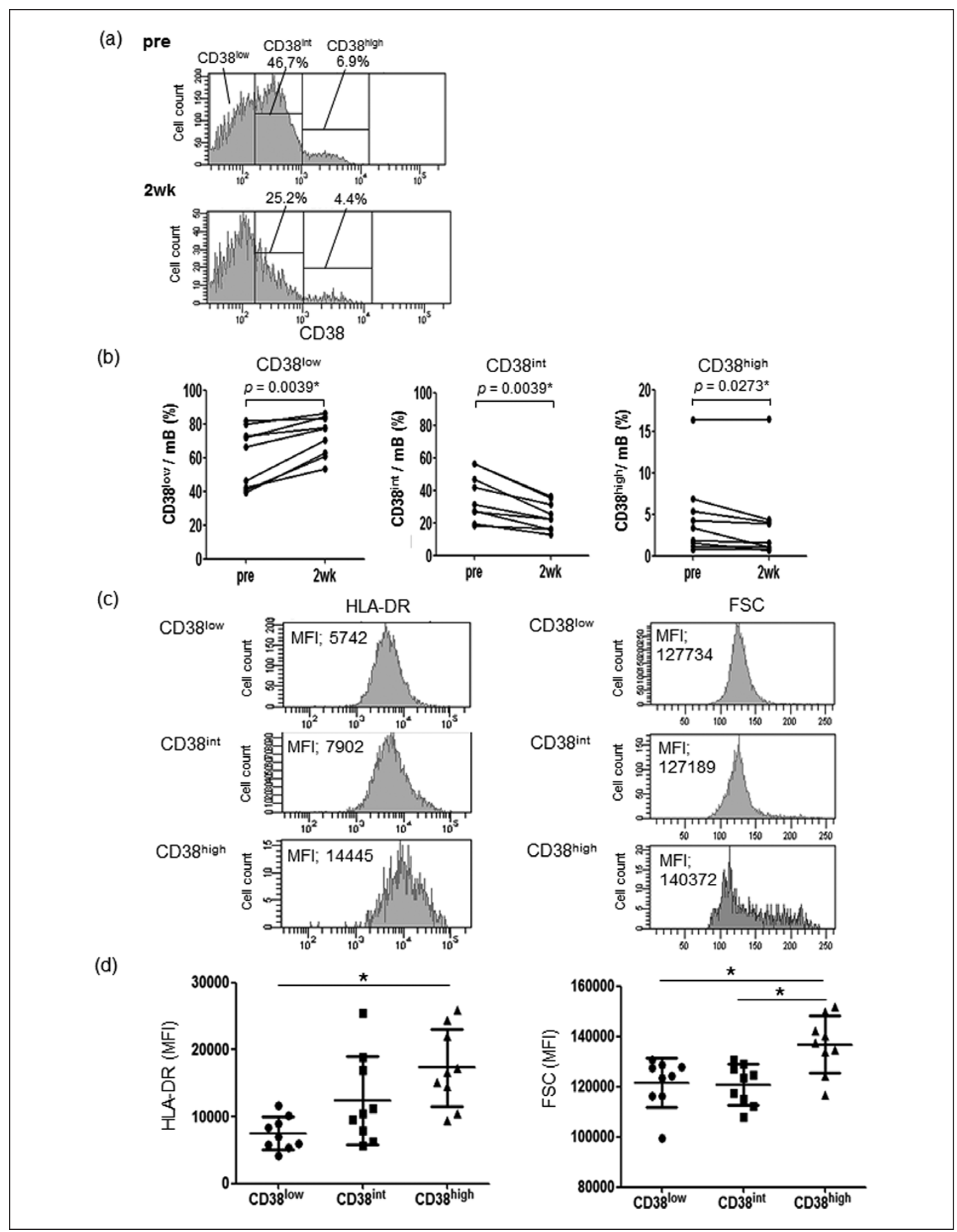

Figure 2. Frequency and activation state of each $\mathrm{mB}$ subpopulation in the peripheral blood of MS patients.

(a)Representative histograms of CD38 expression in mB of peripheral blood from a fingolimod-treated patient. Upper (pre) and lower (2wk) panels show the histograms before and 2 weeks after fingolimod initiation, respectively. The two values above each histogram indicate frequencies of the $\mathrm{mB}$ subpopulations with intermediate (CD38 int, left) and high (CD38 high, right) CD38 expression. (b) Data shown are frequencies of mB subpopulations, classified by CD38 expression levels (CD38 low (left panel), CD38 int (middle panel) and CD38 high (right panel)), in the peripheral blood from nine patients with MS, before (pre) and 2 weeks after ( $2 w k$ ) fingolimod initiation. Data from the same patients are connected with lines. $* p<0.05$ by Wilcoxon signed-rank test. (c) Representative histograms of HLA-DR (left column) and FSC (right column) expression in each mB subpopulation (CD38 low (upper row), CD38 int (middle row) and CD38 high (lower row)) of peripheral blood from a patient with MS, before fingolimod initiation. Values represent MFIs of HLA-DR and FSC. (d) Data shown are MFI of HLA-DR (left panel) and FSC (right panel) in mB subpopulations (CD38low, $C D 38^{\text {int }}$ and $C D 38^{\text {high }}$ ) of peripheral blood from nine patients with MS, before fingolimod treatment. Data are represented as mean \pm SD. $* p<0.05$ by one-way ANOVA and post hoc Tukey's test.

ANOVA: analysis of variance; FSC: forward scatter; HLA: human leukocyte antigen; mB: memory B cells; MFI: mean fluorescence intensity; MS: multiple sclerosis; pre: before treatment; 2 wk: 2 weeks after treatment initiation 
(a)
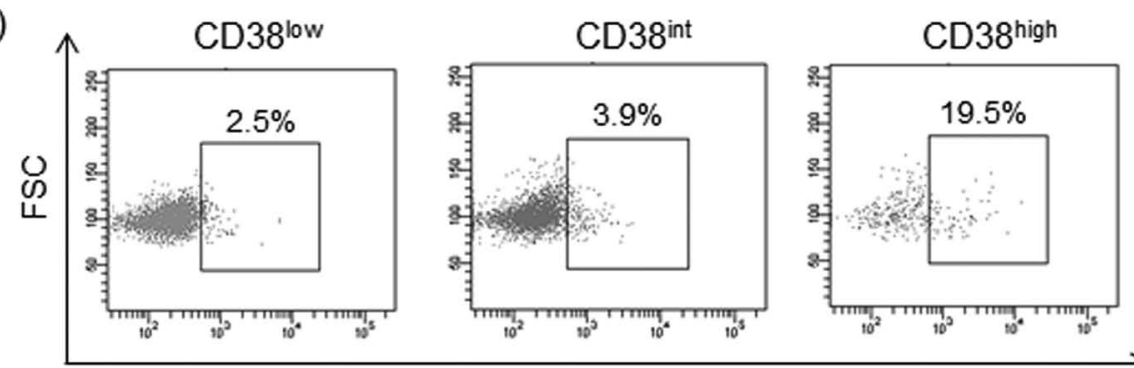

Ki-67

(b)

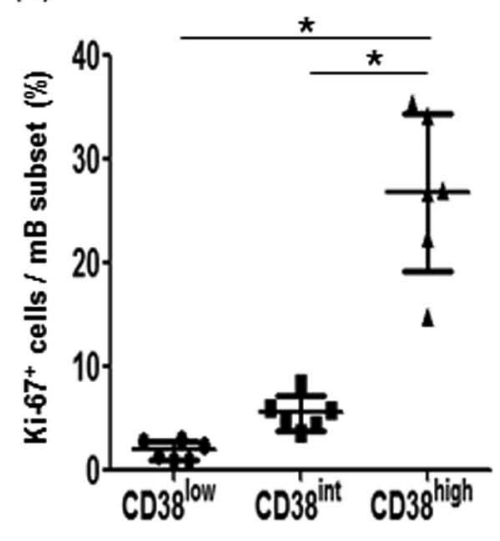

(c)

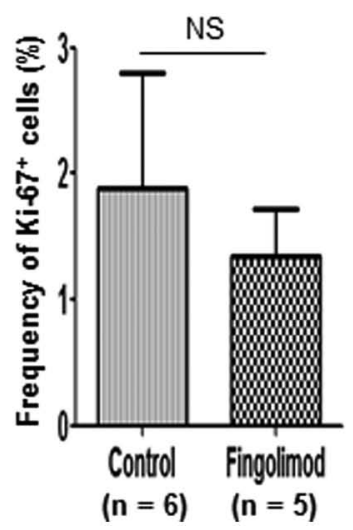

CD38int

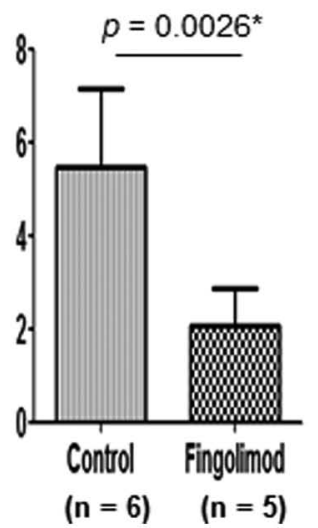

CD38 high

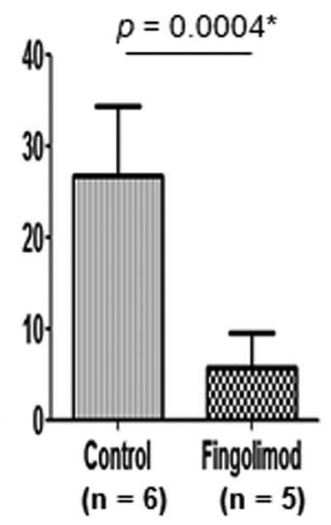

Figure 3. Ki-67 expression in $\mathrm{mB}$ subpopulations of peripheral blood from MS patients.

(a)Representative flow cytometry analyses of intracellular Ki-67 expression in mB subpopulations (CD38 low (left panel), CD38int (middle panel), and CD38high (right panel)) of peripheral blood from an untreated patient with MS. Each mB subpopulation was analysed for FSC and Ki-67 expression. Values in each plot represent frequency of $\mathrm{Ki}-67^{+}$cells in each $\mathrm{mB}$ subpopulation. (b) Frequency of $\mathrm{Ki}-67^{+}$cells in each mB subpopulation of peripheral blood from six untreated patients with MS. Data are represented as mean \pm SD. ${ }^{*} p<0.05$ by one-way ANOVA and post hoc Tukey's test. (c) Frequency of the Ki-67+ population in each $\mathrm{mB}$ subpopulation (CD38 low (left panel), CD38 int (middle panel), and CD38 high (right panel)) is compared between untreated patients with MS (control; $n=6$ ) and fingolimod-treated patients with MS (Fingolimod; $n=5$ ). Mean duration with fingolimod treatment \pm SD is $15.8 \pm 8.8$ (6 to 30 ) weeks. Data are represented as mean \pm SD.

$*_{p}<0.05$ by unpaired $t$-test.

FSC: forward scatter; Ki-67: a marker present only during cell growth or proliferation; mB: memory B cells; MS: multiple sclerosis; NS: not statistically significant.

affecting the total numbers of monocytes and natural killer (NK) cells. ${ }^{16,28,29}$ Furthermore, in MS, fingolimod selectively reduces naïve $\mathrm{T}$ cells, as well as $\mathrm{CD}^{+}$central memory $\mathrm{T}$ cells that are enriched for Th17 cells. ${ }^{6,30} \mathrm{In}$ addition, fingolimod treatment may induce a relative increase in $\mathrm{CD} 27^{-} \mathrm{CD} 28^{-} \mathrm{CD} 8^{+} \mathrm{T}^{-}$cells $^{31}$ and a decrease in CD56 ${ }^{\text {bright }}{ }^{\text {CD62 }} \mathrm{L}^{+} \mathrm{CCR}^{+} \mathrm{NK}$ cells. ${ }^{32}$

The role of autoreactive $\mathrm{CD} 4^{+} \mathrm{T}$ cells in MS pathogenesis has been emphasised over decades. ${ }^{33}$ In contrast, B-cell involvement in MS was highlighted lately, after the clinical effectiveness of rituximab was demonstrated in RRMS patients. Rituximab's effectiveness in MS may result from the depletion of autoantibody-producing B cells, but it can also be explained by depletion of B cells that are able to induce or support activation of autoreactive
T cells. ${ }^{15}$ In fact, B cells exhibit the ability to present antigen to $\mathrm{T}$ cells, and $\mathrm{mBs}$ are more capable than $\mathrm{nBs}$ of supporting the proliferation of neuroantigen-specific $\mathrm{CD} 4^{+} \mathrm{T}$ cells, in vitro. ${ }^{23}$ The presence of oligoclonal bands in the CSF suggests local production of antibodies within the CNS. ${ }^{34}$ Consistent with this, brain lesions ${ }^{13}$ and $\mathrm{CSF}^{14}$ of patients with MS contain clonally-expanded B cells. These results collectively support the postulate that $\mathrm{mBs}$ can potentially trigger the inflammation of MS, either via autoantibody production or via autoantigen presentation to autoreactive T cells.

The focus of this study is to investigate the alterations of peripheral blood B-cell types in fingolimod-treated patients with RRMS. We showed that activated CD38 int and $\mathrm{CD} 38^{\text {high }} \mathrm{mB}$ subpopulations were highly susceptible to 
(a)

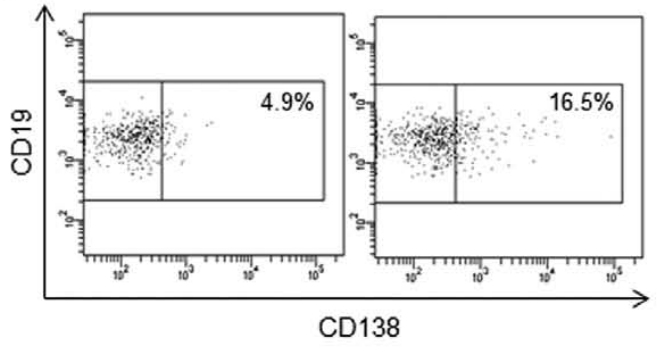

(c)

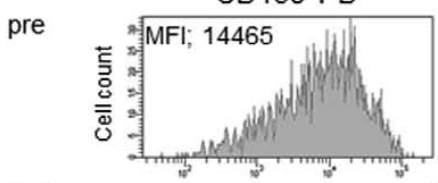

$2 w k$

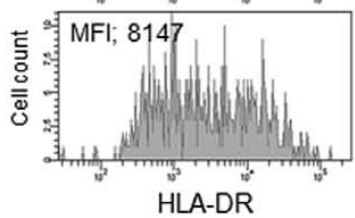

(d)

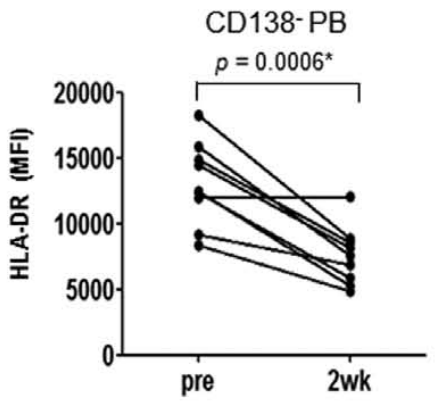

(e)

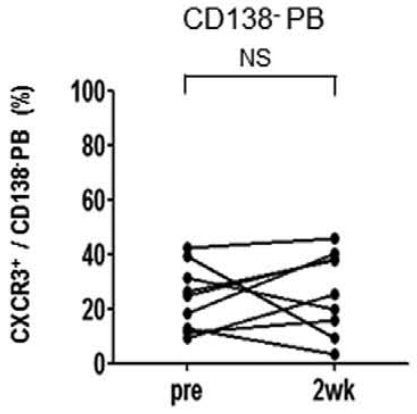

(b)

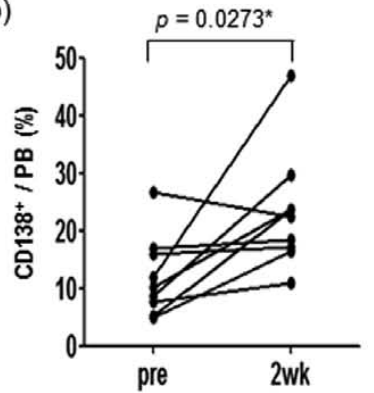

$\mathrm{CD} 138+\mathrm{PB}$
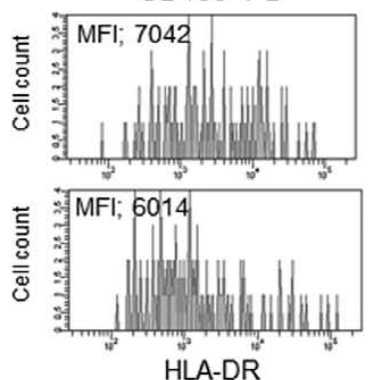

HLA-DR

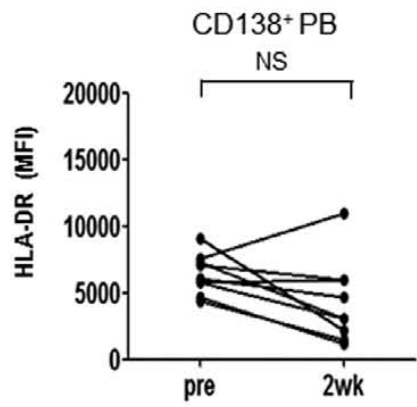

$\mathrm{CD} 138+\mathrm{PB}$

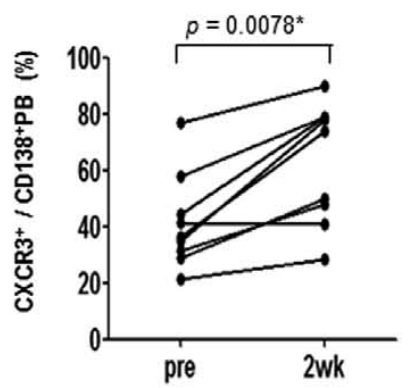

Figure 4. Phenotypic alteration of the remaining $\mathrm{PBs}$ in peripheral blood following fingolimod treatment.

(a) Representative dot plots of CD 19+CD27+CD $80^{-}$CD $38^{\text {high }} \mathrm{PB}$, analysed for CD I 9 and CD 38 expression before (pre) and 2 weeks after ( $2 \mathrm{wk}$ ) fingolimod initiation. Values represent frequencies of the $C D / 38^{+}$subpopulation in total PB. (b) Data are frequencies of the CDI $38^{+}$subpopulation in total PB of peripheral blood from nine patients with MS before (pre) and 2 weeks after ( $2 w k$ ) fingolimod initiation. Data from the same patients are connected with lines. ${ }^{*} p<0.05$ by Wilcoxon signed-rank test. (c) Data are representative histograms of HLA-DR expression in CDI38- and $\mathrm{CDI} 38^{+} \mathrm{PB}$ of peripheral blood, from a patient with MS before (pre) and 2 weeks after ( $2 \mathrm{wk}$ ) fingolimod initiation. Values represent MFI of HLADR. (d) Data are MFI of HLA-DR in CDI38- and CDI38+ PB of peripheral blood from nine patients with MS, before (pre) and 2 weeks after ( 2 wk) fingolimod initiation. Data from the same patients are connected with lines. ${ }^{*} p<0.05$ by paired $t$-test. (e) Data are frequencies of $C X C R 3^{+}$cells in CDI38- PB and CDI38+ PB of peripheral blood from nine patients with MS before (pre) and 2 weeks after (2wk) fingolimod initiation. Data from the same patients are connected with lines. $* p<0.05$ by Wilcoxon signed-rank test.

MFI: mean fluorescence intensity; MS: multiple sclerosis; NS: not statistically significant; PB: plasmablast; pre: before treatment; 2 wk: after 2 weeks of treatment 
fingolimod, as indicated by their reduction in the peripheral blood following fingolimod treatment. It is demonstrated in mice that surface expression levels of S1P1 on B cells in the SLT are controlled by transcription levels and CD69mediated internalisation of S1P1. Stimulation of B-cell receptors induces not only a cessation of S1P1 transcription, but also an upregulation of CD69. Both of these changes reduce the expression levels of surface S1P1 in the SLT to some extent. ${ }^{2}$

Although we were not able to directly analyse B cells in the SLT of the patients, we speculated that surface S1P1 expression on mBs within the SLT in human may also decrease greatly, following antigen activation and exposure to fingolimod, which would result in these B lymphocytes having a reduced responsiveness to S1P. In fact, the activated $\mathrm{mB}$ subpopulations that we isolated from the patients' peripheral blood, in particular CD38 high $\mathrm{mB}$, were found to contain a substantial proportion of $\mathrm{Ki}-67^{+}$ cells (Figure 3(a) and (b)). We confirmed that the proportions of $\mathrm{Ki}-67^{+}$cells in the activated CD38 ${ }^{\text {int }}$ and $\mathrm{CD} 38^{\text {high }}$ $\mathrm{mB}$ subpopulations were significantly decreased following fingolimod treatment, suggesting that recentlyactivated cells were selectively trapped in the SLT following fingolimod treatment. Because activation of autoreactive $\mathrm{mBs}$ in the SLT followed by their migration to the CNS could trigger a relapse of RRMS, ${ }^{35}$ we assumed that inhibition of activated $\mathrm{mB}$ cell egress from the SLT was at least partly involved in the reduced relapses of RRMS after fingolimod treatment.

We also identified a PB subpopulation that is relatively resistant to fingolimod as being $\mathrm{CD} 138^{+} \mathrm{PBs}$. The frequency of the $\mathrm{CD} 138^{+}$subpopulation in the total PBs, and that of $\mathrm{CXCR}^{+}$cells in $\mathrm{CD} 138^{+} \mathrm{PBs}$, was significantly increased by fingolimod treatment. Of note, the $\mathrm{CD} 138^{+} \mathrm{CXCR}^{+} \mathrm{PBs}$ are enriched in the CSF of NMO during relapse, ${ }^{27}$ and fingolimod could induce exacerbation of NMO, accompanied by the appearance of large brain lesions. ${ }^{11,12}$ Although knowledge on the biology of PBs is limited, the percentages of $\mathrm{CCR} 7^{+}$cells are much lower as compared with $\mathrm{nBs}$ or $\mathrm{mBs}$, indicating that fingolimod may differentially alter the in vivo migration of PBs and other B cells.

It is of relevance to note that despite reductions of circulating lymphocytes, RRMS patients receiving fingolimod may develop clinical relapses. These relapses are not always mild, but could be serious and accompany huge brain lesions. ${ }^{7-10}$ Although the trapping of regulatory lymphocytes in the $\mathrm{SLT}^{8,9}$ or the enrichment for CD45RO-CCR7-CD8 ${ }^{+} \mathrm{T}$ cells in the $\mathrm{CSF}^{7}$ is proposed as a possible mechanism for formation of tumefactive brain lesions, we were very curious to know if the increased proportion of $\mathrm{CD} 138^{+} \mathrm{PBs}$ over other lymphocytes in the peripheral blood might influence the character of the CNS pathology and induce large demyelinating lesions. In fact, it was recently reported that $\mathrm{CD} 45^{+} \mathrm{CD} 19^{+} \mathrm{CD} 138^{+} \mathrm{PBs}$ are relatively enriched in the CSF of fingolimod-treated MS patients, ${ }^{16}$ raising the possibility that the dominance of $\mathrm{CD} 138^{+} \mathrm{PBs}$ in the peripheral blood is preserved or even promoted in the CNS of patients with MS who develop tumefactive brain lesions ${ }^{7-10}$ and NMO patients who deteriorate ${ }^{11,12}$ after being treated with fingolimod. Therefore, resistance of activated PBs in fingolimodtreated patients with MS or NMO may give us a clue to understanding the individual patients' differences regarding the effectiveness of fingolimod therapy.

\section{Acknowledgements}

We thank Toshiyuki Takahashi at the Department of Neurology, Tohoku University, for examining serum anti-AQP4-Abs in our patients. We also thank Hiromi Yamaguchi, Yasuko Hirakawa, and Tomoko Ozawa for their technical support.

\section{Conflict of interest}

The authors declare that there are no conflicts of interest.

\section{Funding}

This work was supported by the Ministry of Health, Labour and Welfare of Japan (grant on intractable neuroimmunological diseases number H23-nanchi-ippan-017); and the Japanese Society for the Promotion of Science (grant number: S24229006).

\section{References}

1. Kivisakk P, Mahad DJ, Callahan MK, et al. Expression of CCR7 in multiple sclerosis: Implications for CNS immunity. Ann Neurol 2004; 55: 627-638.

2. Cyster JG and Schwab SR. Sphingosine-1-phosphate and lymphocyte egress from lymphoid organs. Ann Rev Immunol 2012; 30: 69-94.

3. Cohen JA and Chun J. Mechanisms of fingolimod's efficacy and adverse effects in multiple sclerosis. Ann Neurol 2011; 69: 759-777.

4. Kappos L, Radue EW, O'Connor P, et al. A placebo-controlled trial of oral fingolimod in relapsing multiple sclerosis. N Engl J Med 2010; 362: 387-401.

5. Cohen JA, Barkhof F, Comi G, et al. Oral fingolimod or intramuscular interferon for relapsing multiple sclerosis. $N$ Engl $J$ Med 2010; 362: 402-415.

6. Mehling M, Lindberg R, Raulf F, et al. Th17 central memory T cells are reduced by FTY720 in patients with multiple sclerosis. Neurology 2010; 75: 403-410.

7. Pilz G, Harrer A, Wipfler P, et al. Tumefactive MS lesions under fingolimod: A case report and literature review. Neurology 2013; 81: 1654-1658.

8. Jander S, Turowski B, Kieseier BC, et al. Emerging tumefactive multiple sclerosis after switching therapy from natalizumab to fingolimod. Mult Scler 2012; 18: 1650-1652.

9. Visser F, Wattjes MP, Pouwels PJ, et al. Tumefactive multiple sclerosis lesions under fingolimod treatment. Neurology 2012; 79: 2000-2003.

10. Leypoldt F, Munchau A, Moeller F, et al. Hemorrhaging focal encephalitis under fingolimod (FTY720) treatment: A case report. Neurology 2009; 72: 1022-1024. 
11. Izaki S, Narukawa S, Kubota A, et al. [A case of neuromyelitis optica spectrum disorder developing a fulminant course with multiple white-matter lesions, following fingolimod treatment]. Rinsho Shinkeigaku 2013; 53: 513-517.

12. Min JH, Kim BJ and Lee KH. Development of extensive brain lesions following fingolimod (FTY720) treatment in a patient with neuromyelitis optica spectrum disorder. Mult Scler 2012; 18: 113-115.

13. Baranzini SE, Jeong MC, Butunoi C, et al. B-cell repertoire diversity and clonal expansion in multiple sclerosis brain lesions. J Immunol 1999; 163: 5133-5144.

14. Qin Y, Duquette P, Zhang Y, et al. Clonal expansion and somatic hypermutation of $\mathrm{V}(\mathrm{H})$ genes of $\mathrm{B}$ cells from cerebrospinal fluid in multiple sclerosis. J Clin Invest 1998; 102: 1045-1050.

15. Hauser SL, Waubant E, Arnold DL, et al. B-cell depletion with rituximab in relapsing-remitting multiple sclerosis. $N$ Engl J Med 2008; 358: 676-688.

16. Kowarik MC, Pellkofer HL, Cepok S, et al. Differential effects of fingolimod (FTY720) on immune cells in the CSF and blood of patients with MS. Neurology 2011; 76: 1214 1221.

17. Polman CH, Reingold SC, Banwell B, et al. Diagnostic criteria for multiple sclerosis: 2010 revisions to the McDonald criteria. Ann Neurol 2011; 69: 292-302.

18. Takahashi T, Fujihara K, Nakashima I, et al. Establishment of a new sensitive assay for anti-human aquaporin- 4 antibody in neuromyelitis optica. Tohoku J Exp Med 2006; 210: 307-313.

19. Chihara N, Aranami T, Sato W, et al. Interleukin 6 signaling promotes anti-aquaporin 4 autoantibody production from plasmablasts in neuromyelitis optica. Proc Natl Acad Sci USA 2011; 108: 3701-3706.

20. Gohda M, Kunisawa J, Miura F, et al. Sphingosine 1-phosphate regulates the egress of IgA plasmablasts from Peyer's patches for intestinal IgA responses. J Immunol 2008; 180: 5335-5343.

21. Kabashima K, Haynes NM, Xu Y, et al. Plasma cell S1P1 expression determines secondary lymphoid organ retention versus bone marrow tropism. J Exp Med 2006; 203: 2683-2690.

22. Corcione A, Casazza S, Ferretti E, et al. Recapitulation of B-cell differentiation in the central nervous system of patients with multiple sclerosis. Proc Natl Acad Sci USA 2004; 101: 11064-11069.

23. Harp CT, Ireland S, Davis LS, et al. Memory B cells from a subset of treatment-naive relapsing-remitting multiple sclerosis patients elicit CD4(+) T-cell proliferation and IFNgamma production in response to myelin basic protein and myelin oligodendrocyte glycoprotein. Eur J Immunol 2010; 40: 2942-2956.

24. Ruffin N, Lantto R, Pensieroso S, et al. Immune activation and increased IL-21R expression are associated with the loss of memory B cells during HIV-1 infection. J Intern Med 2012; 272: 492-503.

25. Gerdes J, Lemke H, Baisch H, et al. Cell cycle analysis of a cell proliferation-associated human nuclear antigen defined by the monoclonal antibody Ki-67. J Immunol 1984; 133: $1710-1715$.

26. Odendahl M, Mei H, Hoyer BF, et al. Generation of migratory antigen-specific plasma blasts and mobilization of resident plasma cells in a secondary immune response. Blood 2005; 105: 1614-1621.

27. Chihara N, Aranami T, Oki S, et al. Plasmablasts as migratory IgG-producing cells in the pathogenesis of neuromyelitis optica. PLoS One 2013; 8: e83036.

28. Budde K, L Schmouder R Nashan B, et al. Pharmacodynamics of single doses of the novel immunosuppressant FTY720 in stable renal transplant patients. Am J Transpl 2003; 3: 846854.

29. Vaessen LM, Van Besouw NM, Mol WM, et al. FTY720 treatment of kidney transplant patients: A differential effect on B cells, naive $\mathrm{T}$ cells, memory $\mathrm{T}$ cells and NK cells. Transpl Immunol 2006; 15: 281-288.

30. Mehling M, Brinkmann V, Antel J, et al. FTY720 therapy exerts differential effects on T-cell subsets in multiple sclerosis. Neurology 2008; 71: 1261-1267.

31. Johnson TA, Lapierre Y, Bar-Or A, et al. Distinct properties of circulating CD8+ T cells in FTY720-treated patients with multiple sclerosis. Arch Neurol 2010; 67: 1449-1455.

32. Johnson TA, Evans BL, Durafourt BA, et al. Reduction of the peripheral blood CD56(bright) NK lymphocyte subset in FTY720-treated multiple sclerosis patients. J Immunol 2011; 187: $570-579$.

33. Nylander A and Hafler DA. Multiple sclerosis. J Clin Invest 2012; 122: 1180-1188.

34. Meinl E, Krumbholz M and Hohlfeld R. B-lineage cells in the inflammatory central nervous system environment: Migration, maintenance, local antibody production and therapeutic modulation. Ann Neurol 2006; 59: 880-892.

35. Von Budingen HC, Bar-Or A and Zamvil SS. B cells in multiple sclerosis: Connecting the dots. Curr Opin Immunol 2011; 23: 713-720. 